\title{
Day-to-day associations between sleep and physical activity: a set of person-specific analyses in adults with overweight and obesity
}

\author{
Guillaume Chevance ${ }^{1}$ (D) Dario Baretta ${ }^{2}$ Ahmed Jérôme Romain ${ }^{3,4}$ • \\ Job G. Godino ${ }^{5,6,7} \cdot$ Paquito Bernard $^{4,8}$
}

Received: 18 February 2021 / Accepted: 9 August 2021 / Published online: 24 August 2021

(C) The Author(s), under exclusive licence to Springer Science+Business Media, LLC, part of Springer Nature 2021

\begin{abstract}
The objective of the present study was to estimate whether physical activity on one day was associated with both sleep quality and quantity the following night and to examine to what extent sleep on one night was associated with physical activity the next day. We collected data from 33 young adults who were overweight or obese and consistently wore a Fitbit Charge 3. A total of 7094 days and nights were analyzed. Person-specific models were conducted to test the bi-directional associations for each participant separately. Results suggest an absence of association between steps and sleep efficiency in the two directions. More heterogeneous results were observed for the association between steps and total sleep time, with 19 participants (58\%) showing a negative association between total sleep time and next day steps, and 9 (27\%) showing a negative association between steps and next day total sleep time.
\end{abstract}

Guillaume Chevance

guillaume.chevance@isglobal.org

1 ISGlobal, Barcelona Institute for Global Health, Carrer del Dr. Aiguader, 88, 08003 Barcelona, Spain

2 Independent Researcher, Geneva, Switzerland

3 École de Kinésiologie Et Des Sciences de L'activité Physique, Faculté de Médecine, Université de Montréal, Montréal, Québec, Canada

4 Research Center, University Institute of Mental Health At Montreal, Montréal, QC, Canada

5 Center for Wireless \& Population Health Systems, University of California, San Diego, USA

6 Exercise and Physical Activity Resource Center, University of California, San Diego, USA

7 Laura Rodriguez Research Institute, Family Health Centers of San Diego, San Diego, USA

8 Department of Physical Activity Sciences, Université du Québec À Montréal, Montréal, Québec, Canada
Taken together, these results suggest a potential conflicting association between total sleep time and physical activity for some participants. Pre- and post-print doi: https://doi. org/10.31236/osf.io/nfjqv; supplemental material: https:// osf.io/y $7 \mathrm{nxg} /$.

Keywords Idiographic analyses - Personalized medicine Precision medicine $\cdot$ Exercise

\section{Introduction}

Along with eating behaviors, physical activity and sleep are central to the prevention and management of obesity (see for seminal studies, Brown et al., 2009; Cappuccio et al., 2008; Fogelholm et al., 2007; Lemmens et al., 2008). Higher physical activity level is associated with weight reduction or maintenance over time (Lee et al., 2010), and poor sleep quality and quantity is associated with increased risks of future body weight and fat gain (Chaput et al., 2008). Although these two behaviors are often studied separately, research has indicated that they might interact with each other in everyday life (Geller et al., 2017). Physical activity has been hypothesized to be associated with improved sleep via several biological pathways (i.e., decreases in insulin resistance and inflammation, regulation of circadian rhythm, see Chennaoui et al., 2015). In return, sleep deprivation has been hypothesized to alter physical performances and increase fatigue during physical activity (Bromley et al., 2012). Given the central role of physical activity and sleep behaviors in obesity management, it is crucial to better understand how these behaviors interact in the daily lives of people who are overweight or obese. Previous research has already explored how self-reported physical activity and sleep interact in 6-month intervals during a weight 
management program (see Chevance, et al., 2020a for an absence of significant associations between sleep and physical activity in this time interval). The present study focuses on the daily associations between the two behaviors (i.e., changes from day-to-day). Gaining a better understanding of the day-to-day temporal dynamics of these two behaviors may be necessary to develop more effective and personalized behavioral interventions for this population. For instance, this might help to leverage synergistic associations between the two behaviors, or in contrast, prevent antagonistic ones.

A recent systematic review and meta-analysis explored the question of whether physical activity on one day was associated with sleep the following night and to what extent sleep on one night was associated with physical activity the following day (Atoui et al., 2021). This review, which included 33 studies conducted in various populations (but none among participants with weight concerns) did not report any clear pattern of associations between several physical activity and sleep outcomes (Atoui et al., 2021). Similarly, another study conducted among adults with overweight or obesity showed no significant day-to-day associations between the two behaviors (measured with an activity monitor) at the group-level (Liao et al., 2020). This previous study reported that (i) total sleep time was not significantly associated with next day minutes of moderate to vigorous physical activity and that (ii) total sleep time was not associated with next day physical activity neither (Liao et al., 2020).

Although it is possible that physical activity and sleep behaviors are not associated at the group and daily level, a previous review on this topic highlighted that studies exploring this question had some statistical and methodological limitations that preclude drawing a definitive conclusion (see Atoui et al., 2021). First, the measurement of physical activity and sleep behaviors should last for longer time periods than what is usually observed in research (e.g., 7 days of accelerometry; e.g., Irish et al., 2014). This would enable the capture of variability over longer time periods and throughout more diverse contexts. Second, a person-specific approach (i.e., testing the associations among each participant separately, instead of pooled together) would help to estimate inter-individual differences in associations, or in other words, explore whether associations could differ from one person to another (Chevance, et al., 2020b; Kwasnicka $\&$ Naughton, 2019). For example, one study investigating the bi-directional associations between stress and physical activity found positive associations between stress and physical activity for some participants while others presented negative associations, no associations, or associations in only one direction (e.g., stress influencing physical activity but not the opposite; see Burg et al., 2017). Third, linear statistical approaches might not be suitable to capture potentially complex patterns of association between physical activity and sleep. Modeling non-linear associations would help capture potential relationships characterized by a "threshold effect", such as the absence of association between sleep and physical activity up to a particular sleep duration threshold (see for example Bernard et al., 2018). Fourth, only one study so far investigated these associations in adults with overweight or obesity and the study only focused on sleep quantity (i.e., total sleep time), whereas indicators of sleep quality might also be relevant to consider (Atoui et al., 2021; Liao et al., 2020).

To build upon previous research, the objective of the present study was to estimate whether physical activity on one day was associated with both sleep quality and quantity the following night, and to determine to what extent sleep on one night was associated with physical activity the next day. We expected the associations between the two behaviors to be highly heterogeneous across participants in terms of direction (i.e., positive versus negative and unidirectional versus bi-directional), strength (i.e., small versus large effect sizes) and form (i.e., linear versus non-linear).

\section{Methods}

\section{Design}

Data were collected as part of an ongoing randomized controlled trial (RCT; ClinicalTrials.gov Identifier: NCT03907462). All relevant ethical regulations have been followed and participants have completed informed consent. The RCT is a 24-month study including $(i)$ a group with activity monitors and connected digital scales, text messaging, and social media targeting weight loss through behavioral changes, (ii) a group with the abovementioned intervention package and additional phone-based health coaching sessions and (iii) a control group with activity monitors and digital scales alone. As the trial is still ongoing, group assignment remained unknown for the present study and was not modelled in our analyses.

\section{Participants}

Inclusion criteria for the RCT included being between 18 and 35 years of age, being affiliated with a university or college in San Diego, CA as a student, faculty, staff member, or alumni and being overweight or obese $(25>=\mathrm{BMI}<40 \mathrm{~kg} /$ $\mathrm{m} 2$ ). Psychiatric and/or medical conditions represented exclusion criteria for the RCT. No participants had nightshift occupational activities. Participants were continuously recruited from April 16th 2019 (i.e., the beginning of the trial) to March 1st 2020 (i.e., before Covid-19 lockdown measures expected to influence physical activity and sleep 
were introduced in San Diego, CA). All the data analyzed as part of this study were collected before March 1st 2020.

In the present study, the analytic sample comprises participants from this RCT who consistently wore the activity monitor for at least 100 days and nights ( 3 months) with less than $5 \%$ of missing observations for both behaviors (data collection occurred in the first months of the RCT). This cutoff ensures sufficient statistical power at the individual level to test the person-specific associations (i.e., build a statistical model for each participant separately; Ariens et al., 2020).

\section{Measures}

Data on physical activity and sleep were collected with a Fitbit Charge 3 (Fitbit Inc, San Francisco, CA, USA). Participants were asked to wear the wrist-worn device continuously during the trial according to manufacturer recommendations (i.e., to wear the device on one's non-dominant arm and up to three finger widths above the wrist bone). Wear time was assessed in the present analyses using heart rate signals at the minute level. Valid wear time was defined as $1400 \mathrm{~min}$ (i.e., $23 \mathrm{~h}$ and $20 \mathrm{~min}$ ) of existing heart rate signals per day. This constitutes a rather strict threshold, that is justified by the purpose of our study focusing on 24-h behaviors.

Daily step counts were estimated from the Fitbit via triaxial accelerometry. This indicator of physical activity (i.e., step counts) was preferred over other metrics offered by the device (e.g., physical activity intensity) because the estimation of steps from Fitbit sensors range from good to excellent in comparison with direct observation (Feehan et al., 2018), while the validity of other metrics remains open to investigation (O'Driscoll et al., 2018).

Sleep outcomes were estimated via combined accelerometry, heart rate, and heart rate variability signals via Fitbit's proprietary algorithm (see de Zambotti et al., 2018). A previous study showed that the Fitbit Charge accurately discriminated sleep-wake states against polysomnography (de Zambotti et al., 2018). Two sleep outcomes were used: total sleep time and sleep efficiency. Total sleep time was directly estimated by the device, whereas sleep efficiency was computed as follows: [total sleep time / total time in bed $(\times 100)]$ (Reed \& Sacco, 2016). Total time in bed was estimated from Fitbit's proprietary algorithm (to our knowledge, the algorithm was updated in 2017, and should have remain the same during our data collection period).

With regards to descriptive statistics, weight and height were measured objectively and used to compute participants' BMI in $\mathrm{kg} / \mathrm{m}^{2}$. Age and sex were self-reported.

Variables known to potentially affect the association between physical activity and sleep behaviors were controlled for (i.e., public holidays, week-days versus weekenddays, and seasons of the year; see for instance Matthews et al., 2002; Mattingly et al., 2021). These indicators were created based on the internal date recorded by the Fitbit. We also controlled for naps (see the statistical section below).

\section{Statistical analyses}

This study adopted a person-specific (i.e., idiographic) approach, meaning that each participant represented an analytical unit and was analyzed separately from the others. In other words, participants were not pooled together. This is different from what it is usually done at the group-level (i.e., nomothetic research, see Chevance, et al., 2020b; Kwasnicka \& Naughton, 2019).

Linear regression models were used to fit to the data for each participant separately (Vieira et al., 2017). Four models were specified (see Table 1). The first model included the daily number of steps as a dependent variable and total sleep time the previous night as the independent variable. The second included steps as a dependent variable and sleep efficiency the previous night as the independent variable. The third included total sleep time as a dependent variable and steps the day before as the independent variable. The fourth included sleep efficiency as a dependent variable and steps the day before as the independent.

Each model included the following control variables: (i) both the dependent and independent variables at the

Table 1 Models specifications

\begin{tabular}{|c|c|c|c|c|}
\hline Models & $\begin{array}{l}\text { Dependent variable } \\
(\text { time } t+1)\end{array}$ & $\begin{array}{l}\text { Independent variable } \\
\text { (time t) }\end{array}$ & $\begin{array}{l}\text { Control variables } \\
\text { (previous lags for each } \\
\text { variable) }\end{array}$ & Additional control variables \\
\hline Model 1 & Steps & Total sleep time & $\begin{array}{l}\text { Steps } \\
\text { Total sleep time }\end{array}$ & \multirow{4}{*}{$\begin{array}{l}\text { Progressive day } \\
\text { Season of the year } \\
\text { Holiday } \\
\text { Weekend } \\
\text { Nap occurrence }\end{array}$} \\
\hline Model 2 & Steps & Sleep efficiency & $\begin{array}{l}\text { Steps } \\
\text { Sleep efficiency }\end{array}$ & \\
\hline Model 3 & Total sleep time & Steps & $\begin{array}{l}\text { Total sleep time } \\
\text { Steps }\end{array}$ & \\
\hline Model 4 & Sleep efficiency & Steps & $\begin{array}{l}\text { Sleep efficiency } \\
\text { Steps }\end{array}$ & \\
\hline
\end{tabular}


previous lag, to control for potential auto-correlations; (ii) the progressive number of days in the study, to control for the effect of time on the associations; (iii) a set of control variables, including the seasons (4 binary variables for each season), holidays (a binary variable), the distinction between week-days and weekends (a binary variable), as well as naps (binary outcome: $0=$ no sleep records detected by the device during the day versus $1=$ one or more sleep records detected by the device during the day).

As our hypotheses also included the possibility of nonlinear relationships, we fitted Generalized Additive Models (GAMs; Hastie \& Tibshirani, 1986) for each of the four above-mentioned models. GAMs is a method that has been previously recommended for analyzing person-specific time series (Shadish et al., 2014). It allows the relationships between the independent and dependent variables to be modelled by smooth curves, thus providing a tool to model potential non-linearity in an additive structure. The four GAMs were conducted with a similar structure to the linear models but included one-dimensional smoother terms (f) for the independent variables to discover their functional forms (please see the $\mathrm{R}$ code in supplemental materials for models' specifications, https://osf.io/y $7 \mathrm{nxg} /$ ).

Both the linear and generalized additive models were compared using Akaike's Information Criterion (AIC), with smaller values indicating a better fit (Vrieze, 2012). GAMs estimate non-linearity using the effective degrees of freedom (edf) of the smoothing terms, with edf $>3$ indicating form of non-linearity (Shadish et al., 2014).

No imputations were applied because our inclusion criteria included less than 5\% of missing data for steps and sleep behaviors (i.e., patterns of missing data were visually inspected to ensure that they were distributed relatively consistently across the time-series; a criterion that was met). Outlier values were visually inspected before running the models (no observations were removed). Given the distribution of our dependent variables and after having checked potential trends in the time-series, we decided not to perform any transformations before conducting the analyses (see supplemental materials \#1 and \#5 at https://osf.io/y7nxg/).

The packages $m g c v$ (Wood, 2017) and visreg (Breheny \& Burchett, 2017) from the from the statistical software R ( R version 4.0.2; $R$ Core Team, 2017) were used to conduct and visualize the models. The data and code used for the main analyses, as well as supplemental materials are provided on the Open Science Framework repository platform (see https://osf.io/y7nxg/).

\section{Results}

\section{Participants and descriptive statistics}

A total of 33 participants and 7094 days and nights were analyzed [participants' characteristics: $61 \%$ female; mean age $=26$ years (range $=19-36)$, mean BMI at baseline $=30 \mathrm{~kg} / \mathrm{m} 2($ range $=25-36)]$. Participants were mostly white or Caucasian $(56 \%$; $41 \%$ indicated an Hispanic or Latino origin), incomes over the last 12-months were fairly distributed between 16,000 and 75,000 USD, and most participants reported some college or associate degree as their highest level of education.

On average, participants wore their device for 215 days (range $=106-304$; note that the recruitment for this trial started on April 16th, 2019 and is still ongoing; given that we only selected pre-pandemic data, this gives a potential time-series length comprised of between 100 and 320 days corresponding to the beginning of the trial). The average number of daily steps across the 33 participants was 9,848 steps per day (range $=4,370-16,092$ ), average total sleep time was 408 min per night ( $\sim$ roughly $7 \mathrm{~h}$; range $=328-488$ ) and average sleep efficiency was $87 \%$ (range $=84-90$ ). Descriptive information for each participant is provided as supplemental material (see supplemental material \#2). These 33 participants were selected out of 345 participants with at least 100 days of observation during the period considered. These 33 participants did not visually differ from the excluded participant in regards of each outcomes of interest (see supplemental material \#6 at https://osf.io/y $7 \mathrm{nxg} /$ ). The time series below provide an illustration of the three outcomes over time for two participants (\#310, left panel and \#246, right panel; see Fig. 1).

\section{Testing potential non-linearities}

Overall, AIC were comparable between participants for the linear and GAMs models, meaning that the addition of the smoothed (i.e., non-linear) term did not drastically improve model fit, nor did it deteriorate model fit (see supplemental material \#3 for each participant model's outcome at https:// osf.io/y7nxg/). The effective degrees of freedom (edf) of the smoothing term were often around 1.0 and rarely above 3.0 (i.e., 28 times over 132 tested models; 20\%), indicating an overall linear trend between steps, total sleep time, and sleep efficiency. Although, some edfs values were above 3, for 5-9 participants depending on the specific associations, visual inspections of the plots revealed that the apparent non-linearities were mainly driven by artifacts (see supplemental material \#4 for the plots of each participants' GAMs). Given the non-superiority of the GAMs over the linear models, only the linear models are presented in the following section. 

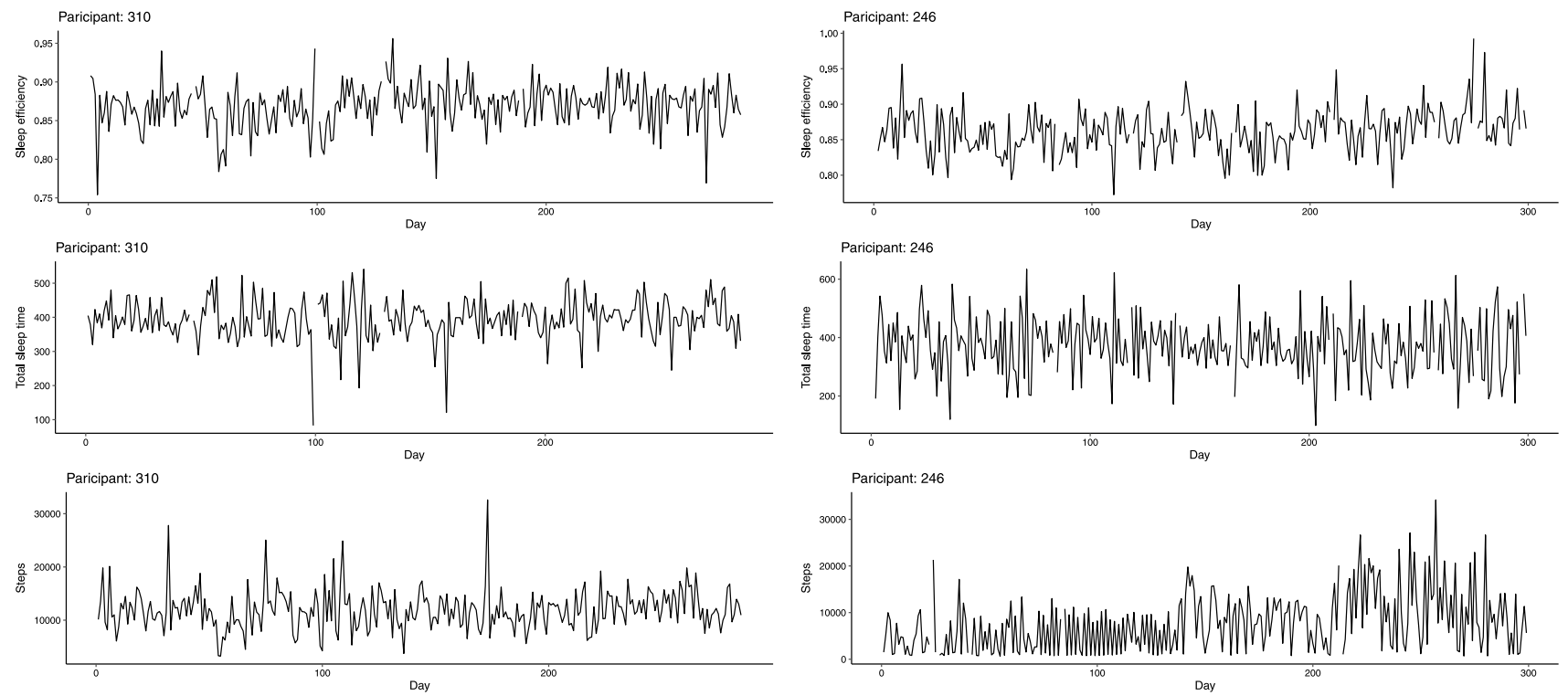

Fig. 1 Illustration of the three study outcomes for 2 participants. See all the time-series analyzed in this study in supplemental material \#5 (https://osf.io/y7nxg/)

We decided to present the outcomes of the linear models (i.e., estimate of each association with their $95 \%$ confidence intervals) with forest plots, in line with person-specific/idiographic analyses reporting practices (Burg et al., 2017). Each line of the forest plot represents one participant's estimate with its respective confidence intervals. To ease the interpretation of the results, forest plots are displayed separately for the participants presenting respectively an average sleep time lower and higher than $7 \mathrm{~h}$ per night ( $7 \mathrm{~h}$ of sleep being usually considered as the optimal sleep duration at the population level, thus being potentially important to control for when interpreting our results; Chaput et al., 2020).

\section{Associations between total sleep time on one night and steps the following day (model 1)}

Figure 2 shows the forest plots of the person-specific associations between total sleep time on one night and steps the next day. The forest plots highlight that total sleep time was significantly (based on the confidence intervals) and negatively associated with the number of steps the following day for 19 participants out of 33 (58\%), and not significantly associated with steps for the remaining 14 participants. Visually, this pattern of results was relatively similar between the participants sleeping more (upper panel) or less (lower panel) than $7 \mathrm{~h}$ on average per night.

To illustrate, for the participant with the strongest negative association between total sleep time and steps (participant \#1099, last line of the forest plot), sleeping longer on one night was associated with a lower number of steps the following day. Specifically, on average across the study, sleeping one more minute on one day was associated with a mean decrease of 20 steps the following day for that specific participant.

\section{Associations between sleep efficiency on one night and steps the following day (model 2)}

Figure 3 shows the forest plots of the person-specific associations between sleep efficiency on one night and steps the next day. The forest plots highlighted that, for a majority of participants $(n=31 ; 95 \%)$, sleep efficiency was not significantly associated with the number of steps the following day. This association was positive and significant for one participant (\#246), indicating that, for this person specifically, higher sleep efficiency on one night was associated with a higher number of steps the following day. On the contrary, sleep efficiency was significantly and negatively associated with steps for the participant \#742. This pattern of results was similar between the participants sleeping more or less than $7 \mathrm{~h}$ on average per night.

\section{Associations between steps on one day and total sleep time the following night (models 3 )}

Figure 4 shows the forest plots of the person-specific associations between steps on one day and total sleep time the following night. The forest plots highlighted that the number of steps on one day was significantly and negatively associated with total sleep time the following night for 9 participants out of $33(27 \%)$, not-significantly associated for 23 participants (70\%), and positively associated for one participant. 
Average sleeping time $>7$ hours

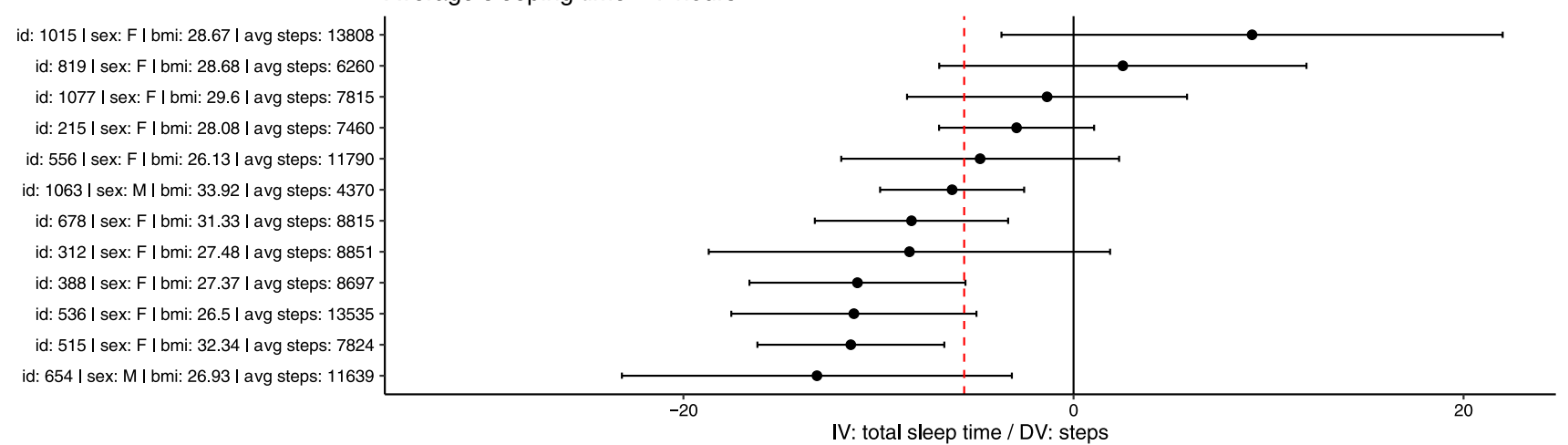

Average sleeping time $<7$ hours

id: 992 | sex: M | bmi: 26.34 | avg steps: 9162

id: 742 | sex: F | bmi: 32.85 I avg steps: 9233 id: 1014 I sex: F | bmi: 35.26 I avg steps: 14064

id: 1129 | sex: M I bmi: 35.06 | avg steps: 8401

id: 321 | sex: M I bmi: 28.98 | avg steps: 6809

id: 335 | sex: F | bmi: 28.93 I avg steps: 9756

id: 193 | sex: M I bmi: 30.06 | avg steps: 8472

id: 621 | sex: M | bmi: 32.81 | avg steps: 6967

id: 667 | sex: M | bmi: 31.22 | avg steps: 9554

id: 771 I sex: F I bmi: 34.7 I avg steps: 8878

id: 878 I sex: M I bmi: 27.02 I avg steps: 10614

id: 344 | sex: F | bmi: 25 I avg steps: 6642

id: 1140 I sex: F I bmi: 34.68 I avg steps: 12047

id: 1111 | sex: F I bmi: 30.18 I avg steps: 6747

id: 296 I sex: F I bmi: 28.15 | avg steps: 11385

id: 310 I sex: F I bmi: 30.19 I avg steps: 11921 .

id: 883 | sex: F | bmi: 33.49 | avg steps: 11700

id: 887 I sex: M I bmi: 35.95 I avg steps: 16092

id: 246 | sex: M | bmi: 31.31 | avg steps: 7626

id: 1073 I sex: M I bmi: 28.58 I avg steps: 13420 .

id: 1099 | sex: M | bmi: 26.7 | avg steps: 14628

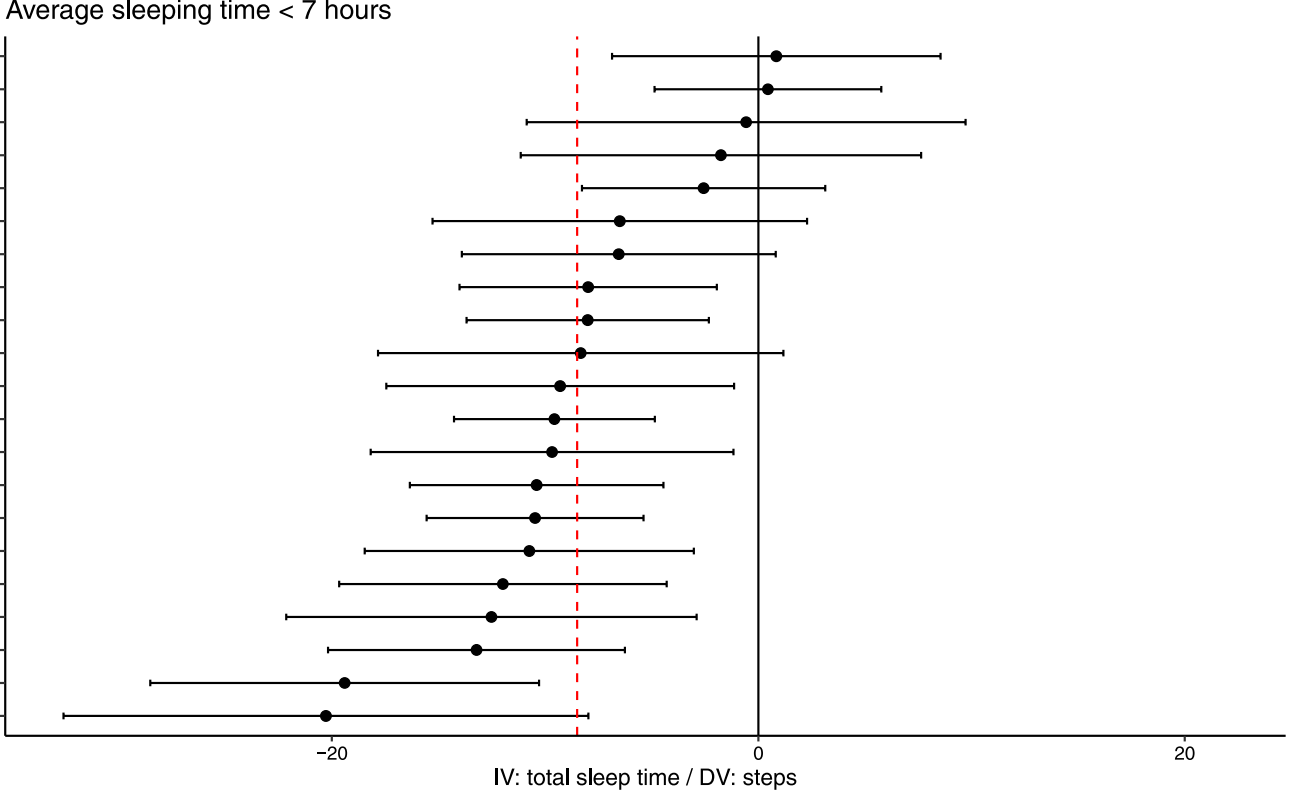

Fig. 2 Associations between total sleep time on one night and steps the next day. IV=independent variable; DV=dependent variable; X- axis represents changes in daily steps. The dashed red line represents the average association across participants

The pattern of results was similar between the participants sleeping more or less than $7 \mathrm{~h}$ on average per night.

\section{Associations between steps on one day and sleep efficiency the following night (models 4)}

Figure 5 shows the forest plots of the person-specific associations between steps on one day and sleep efficiency the following night. The forest plots highlight that the number of steps on one day was not associated with sleep efficiency the following night for all but one participant. For this participant (\#678), more steps than the mean level on one day was associated with higher than usual sleep efficiency. Again, the pattern of results appeared relatively similar between the participants sleeping more or less than $7 \mathrm{~h}$ on average per night.

\section{Summary of the results and bi-directionality}

Results from the four models are summarized in Table 2. Taken together, results suggest an absence of association between steps and sleep efficiency in the two directions for most participants (i.e., these associations were not significant for $94 \%$ and $97 \%$ of the participants). Slightly more heterogeneous results were observed for the association between steps and total sleep time, with $58 \%$ of our participants showing a negative association between total sleep time and next day steps, and $27 \%$ showing a negative association between steps and next day total sleep time.

The association between steps and total sleep time was significant in both directions for 5 participants, thus indicating bi-directional associations. Participants \#536, 515, 344 , and 621 presented a significant and negative association between steps and total sleep time in the two directions. For these participants, doing more steps on one day 
Average sleeping time $>7$ hours

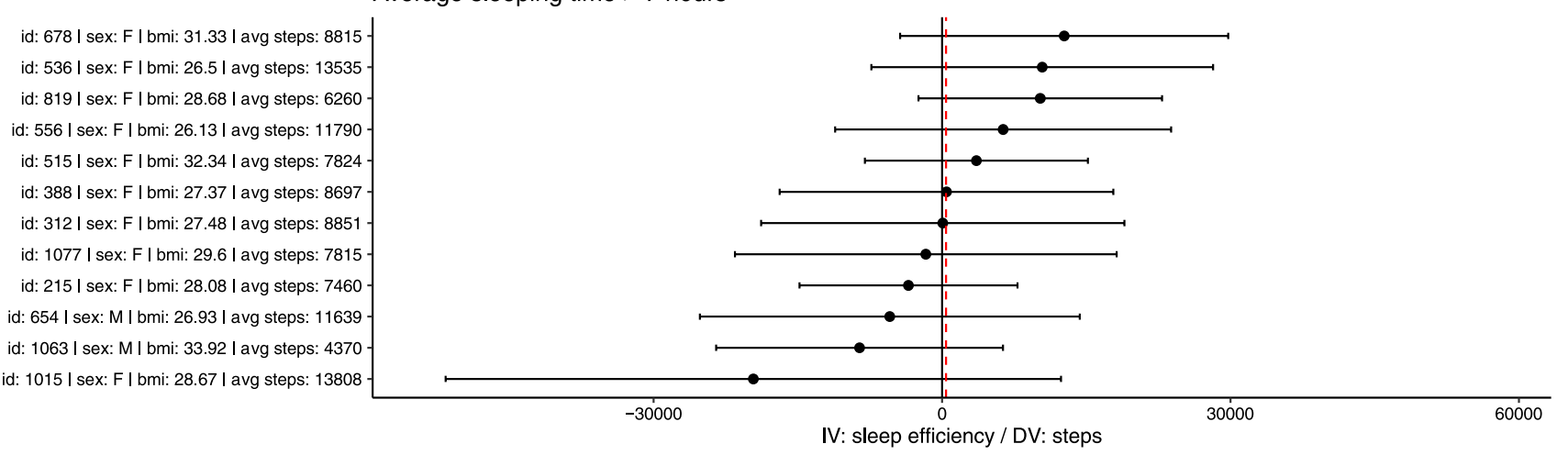

Average sleeping time $<7$ hours

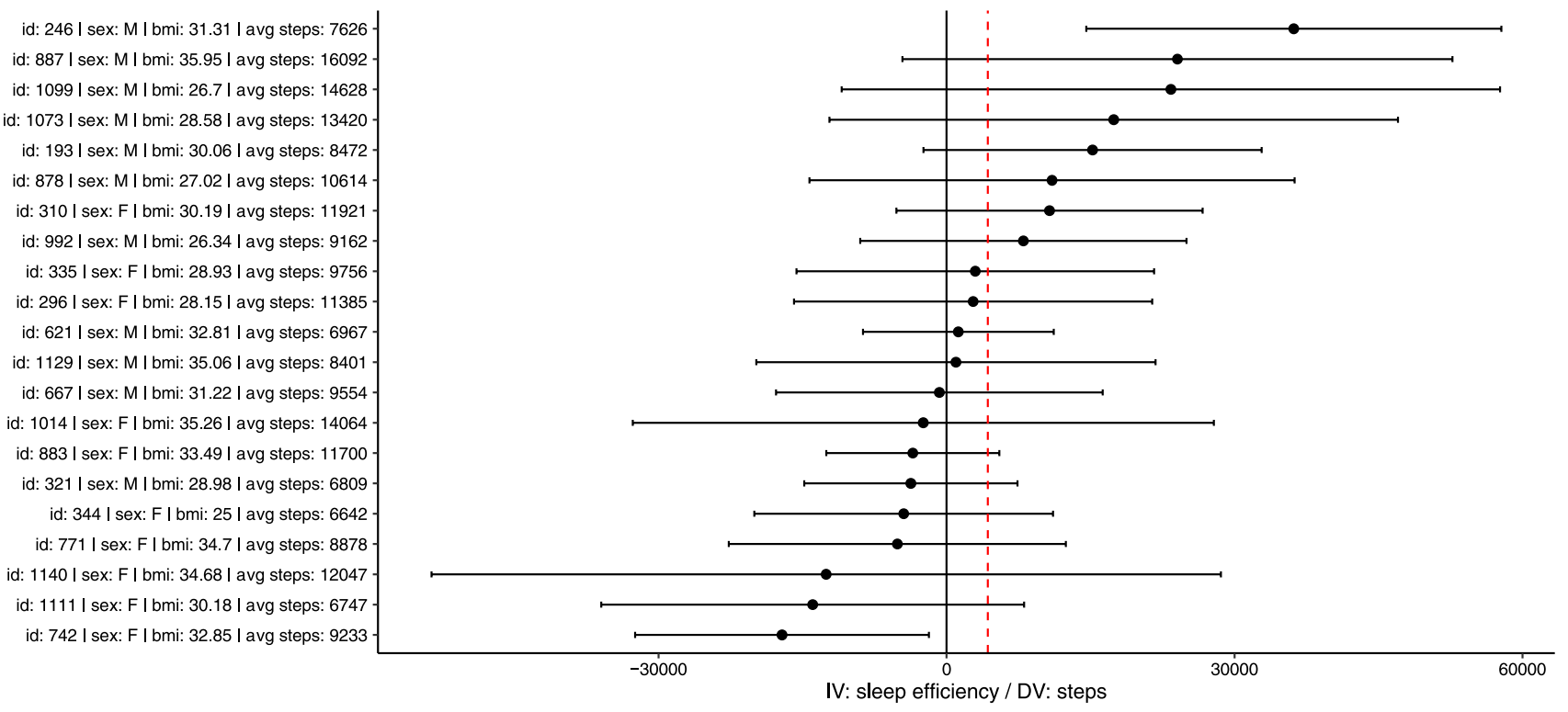

Fig. 3 Associations between sleep efficiency on one night and steps the next day. IV=independent variable; DV=dependent variable; X- axis represents changes in daily steps. The dashed red line represents the average association across participants

was associated with shorter sleep duration the following night, and shorter sleep duration on one night was associated with more steps the following day. One participant (\#246) presented a singular pattern of results: different from the other participants, more steps on one day was associated with longer sleep duration the following night, but, as for the other participants, shorter sleep duration on one night was associated with more steps the following day.

\section{Ancillary analyses}

In order to explore the impact of 7-day lags on associations, we conducted four models (see Table 1) with the independent variables included up to 7-day (instead of the 2-day lags considered in the initial analyses). To ease the interpretation, results are presented with heat maps in supplemental material; see "heat maps" at https://osf.io/y7nxg/).
Taken together, the heat maps clearly suggest that the strongest associations occurred at lag-1, notably for the associations between steps and total sleep time (i.e., model 1 and model 2). Although, significant associations were found for each model after lag-1 and up to lag-7, no clear daily or weekly patterns can be identified from the heat maps and some of these association might be due to hazard (type-1 error). Interestingly, two participants (\#1099 and \#654; those presenting the strongest negative association between total sleep time and next day steps in the model 1) showed persistent significant associations for model 1 at lag-2, lag-3 and up to lag-6. This suggests that these two participants are likely to experience a "negative accumulation effect" of shorter sleep on their physical activity several days after. 
Average sleeping time $>7$ hours

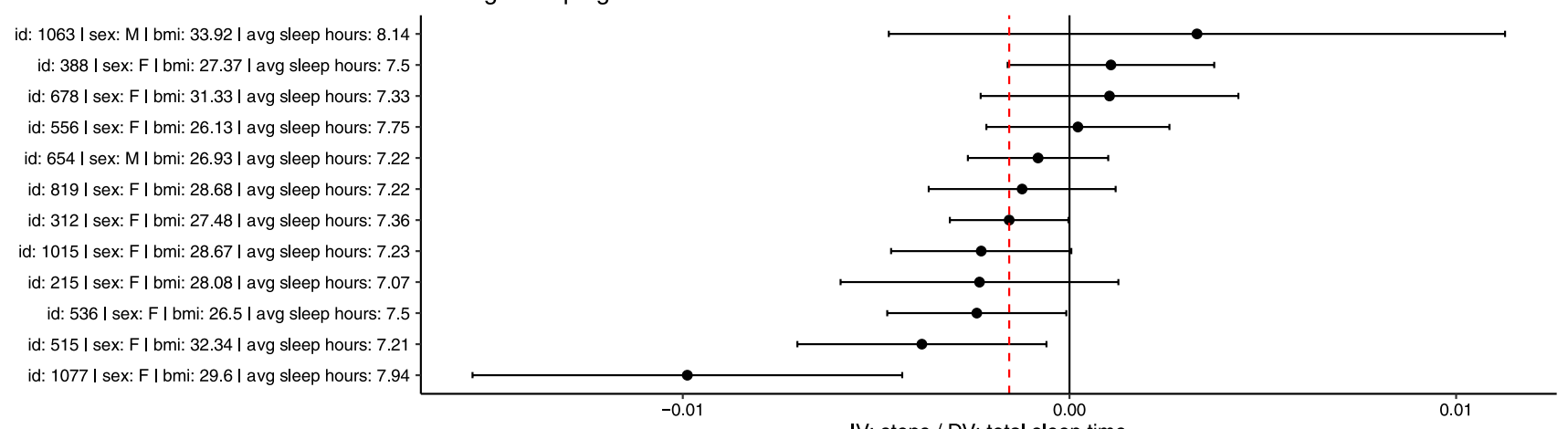

IV: steps / DV: total sleep time

Average sleeping time $<7$ hours

id: 246 | sex: M I bmi: 31.31 | avg sleep hours: 6.2 id: 742 I sex: $F$ | bmi: 32.85 | avg sleep hours: 6.74 id: 1129 | sex: M | bmi: 35.06 | avg sleep hours: 6.7 id: 1099 | sex: M | bmi: 26.7 | avg sleep hours: 6.46 id: 1140 I sex: F | bmi: 34.68 I avg sleep hours: 6.96 id: 878 I sex: M I bmi: 27.02 I avg sleep hours: 6.77 id: 321 I sex: M I bmi: 28.98 I avg sleep hours: 6.22 id: 1111 | sex: F I bmi: 30.18 | avg sleep hours: 6.88 id: 887 I sex: M I bmi: 35.95 I avg sleep hours: 6.42 id: 1014 I sex: F I bmi: 35.26 I avg sleep hours: 5.92 id: 667 | sex: M | bmi: 31.22 | avg sleep hours: 6.9 id: 310 I sex: F I bmi: 30.19 | avg sleep hours: 6.56 id: 883 I sex: F I bmi: 33.49 I avg sleep hours: 6.34 id: 771 | sex: F | bmi: 34.7 I avg sleep hours: 6.23 id: 193 I sex: M I bmi: 30.06 I avg sleep hours: 5.67 id: 335 I sex: $F$ I bmi: 28.93 I avg sleep hours: 6.64 id: 296 I sex: F I bmi: 28.15 | avg sleep hours: 6.79 id: 1073 I sex: M I bmi: 28.58 I avg sleep hours: 5.46 id: 992 I sex: M I bmi: 26.34 I avg sleep hours: 6.57 id: 344 I sex: F I bmi: 25 I avg sleep hours: 6.61 id: 621 I sex: M I bmi: 32.81 I avg sleep hours: 5.84

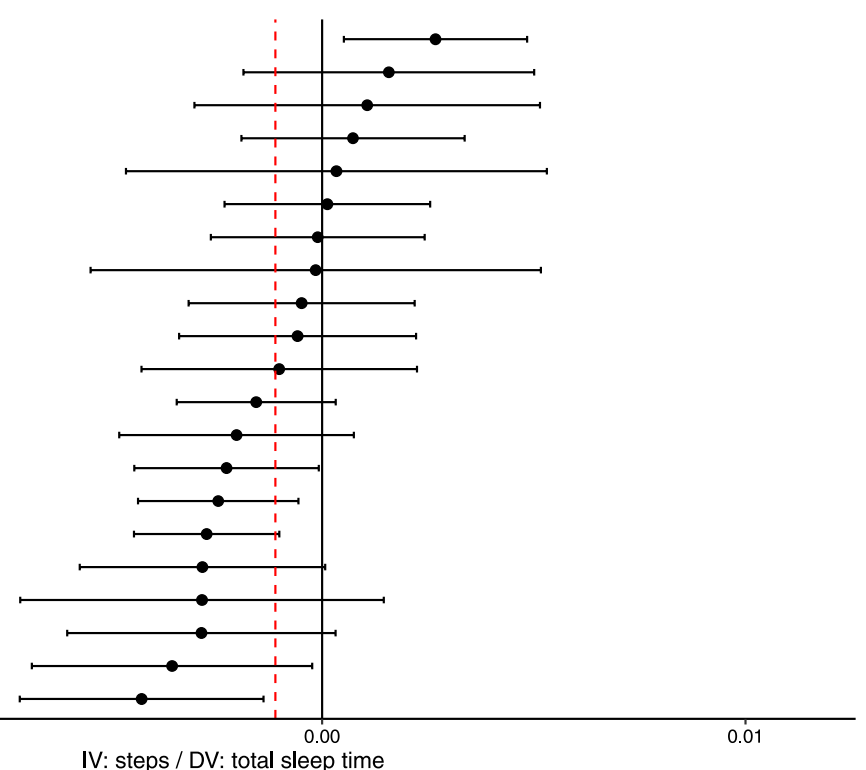

Fig. 4 Associations between steps on one day and total sleep time the following night. IV = independent variable; $\mathrm{DV}=$ dependent variable; $\mathrm{X}$ - axis represents changes in total sleep time. The dashed red line represents the average association across participants. IV =inde-

\section{Discussion}

The study objective was to estimate the day-to-day associations between physical activity (i.e., steps) and sleep quantity (i.e., total sleep time) and quality (i.e., sleep efficiency) over several months among young adults who are overweight or obese. We hypothesized heterogeneous associations across participants in terms of direction, strength, and form. This hypothesis was only partially supported by our results. Although we found some heterogeneity in the directions and strength of these associations, significant results were mostly observed for the associations between steps and total sleep time. This association was mainly unidirectional with sleep being associated with next day steps (model 1; $58 \%$ of the sample), more than the opposite direction (model $3 ; 27 \%$ of the sample). No heterogeneity was observed in the pendent variable; $\mathrm{DV}=$ dependent variable; $\mathrm{X}$ - axis represents changes in sleep efficiency. The dashed red line represents the average association across participants

form of the associations, and our results highlighted linear instead of non-linear associations between the two behaviors at the individual level.

Importantly, we observed a negative association between total sleep time and next day steps for a majority of the participants (58\%; model 1). This is aligned with results from previous research conducted over shorter-time periods of time (i.e., a week) and at the group level (e.g., Baron et al., 2013; Bernard et al., 2016; Fanning et al., 2016; Mead et al., 2019). Among 9 studies included in a recent systematic review that tested this day-to-day association at the withinperson level, 4 reported a non-significant association, 4 a negative association (i.e., the ones just previously cited) and one a positive association. This result thus appears consistent with previous studies. In the opposite direction, the association between steps and sleep duration the following night 


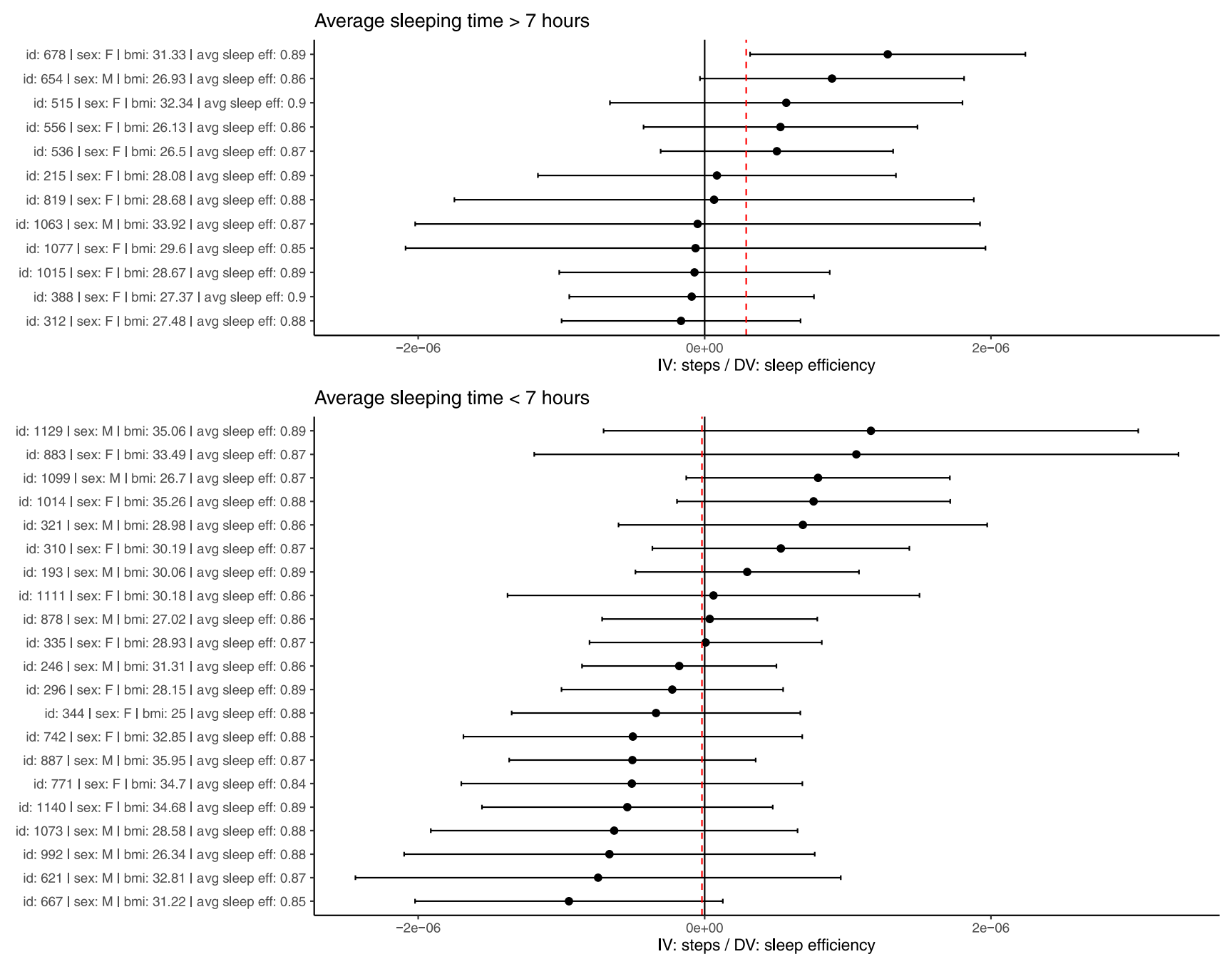

Fig. 5 Associations between steps on one day and sleep efficiency the following night. IV=independent variable; DV = dependent variable; Xaxis represents changes in sleep efficiency. The dashed red line represents the average association across participants.

Table 2 Summary of the results

\begin{tabular}{|c|c|c|c|c|c|c|}
\hline \multirow[b]{2}{*}{ Direction of the association } & \multicolumn{3}{|c|}{ TST $\longrightarrow$ Steps (model 1) } & \multicolumn{3}{|c|}{$\mathrm{SE} \longrightarrow>$ Steps (model 2) } \\
\hline & positive & negative & n.s & positive & negative & n.s \\
\hline \multirow[t]{2}{*}{ Frequency $(\mathrm{N}=33)$} & $\begin{array}{l}0 \\
(0 \%)\end{array}$ & $19(58 \%)$ & $14(42 \%)$ & $\begin{array}{l}1 \\
(3 \%)\end{array}$ & $\begin{array}{l}1 \\
(3 \%)\end{array}$ & $\begin{array}{l}31 \\
(94 \%)\end{array}$ \\
\hline & \multicolumn{3}{|c|}{ Steps $\longrightarrow>$ TST (models 3) } & \multicolumn{3}{|c|}{ Steps $\rightarrow$ SE (models 4) } \\
\hline Direction of the association & positive & negative & n.s & positive & negative & n.s \\
\hline Frequency $(\mathrm{N}=33)$ & $\begin{array}{l}1 \\
(2.5 \%)\end{array}$ & $\begin{array}{l}9 \\
(27 \%)\end{array}$ & $\begin{array}{l}23 \\
(70 \%)\end{array}$ & $\begin{array}{l}1 \\
(3 \%)\end{array}$ & $\begin{array}{l}0 \\
(0 \%)\end{array}$ & $\begin{array}{l}32 \\
(97 \%)\end{array}$ \\
\hline
\end{tabular}

$T S T$, total sleep time; $S E$, sleep efficiency; $n . s$, non-significant

was significant and negative for almost a third of the sample (model 3). This means that for some participants, being more active on one day can also be associated with less sleep the following night. To date, mostly non-significant day-to-day associations between physical activity and sleep duration the following night have been reported in previous studies (11/14 studies in a recent systematic review; see Atoui et al., 2021). This relative discrepancy with previous findings 
could be due to the nature of the idiographic analyses used in the present study, which allow the detection of significant person-specific associations that might not appear at the group level.

From a behavioral perspective, these two negative associations (models 1 and 3) could be interpreted as a negative spillover between steps and sleep duration (Geller et al., 2017). Sleeping longer than usual might create a conflict with physical activity for some participants, leaving less time to be active during the day. Similarly, being more active on one day might trigger shorter sleep duration potentially because these participants have chosen to not adjust their daily routine those days when they were more active than their usual level, thus leaving less time to sleep the following night. This pattern of result is particularly interesting because it underlines the complex, potentially conflicting, nature of multiple behavior change promotion. For example, in a weight management program, interventionists might want to help some individuals schedule their daily routine in a way that supports both physical activity and sleep (i.e., avoids compromising one behavior to the detriment of the other). Because circadian disruption is detrimental for health (Vetter, 2020), we would recommend to not intervene on sleep habits (e.g., asking participants to wake-up earlier or go to bed later to leave more time for being active), at least for individuals like those included in the present study that do not present with any known sleep disorders. Interventions designed to manage goal conflicts (Conner et al., 2016) or leverage behavior change techniques, such as action planning or time management (Knittle et al., 2020), might help participants to schedule their daily routine in a way that support both behaviors (i.e., finding time to be active without hindering sleep). An additional consideration is that the type of physical activity might also explain the individual differences observed in the magnitude of the association between physical activity and sleep duration. For instance, participants engaging in structured activities (i.e., exercise) might be more vulnerable to longer sleep duration (because their planned exercise session might be missed when they have longer sleep than usual) compared to participants that are used to accumulating their physical activity throughout the day, via, for instance, active transportation. Finally, it should be noted that total sleep time captured both $(i)$ available time for physical activity and (ii) "pure" effects of sleep duration. It is possible that the significant associations detected between total sleep time and steps are representative of time constraints more than an effect of shorter/longer sleep. Future studies might explore this question by controlling for wake periods.

In regard to the two other associations tested (models 2 and 4), we mostly found non-significant relationships between steps and sleep efficiency in the two directions (i.e., $94 \%$ and $97 \%$ of the participants; see Table 2). This is in accordance with results from previous studies conducted across other populations that show few significant associations between these two outcomes at the daily level (e.g., Bernard et al., 2016; Mead et al., 2019). Although, physical activity and sleep quality are sometimes presented as positively influencing each other in the short-term (Kredlow et al., 2015; Tang \& Sanborn, 2014), empirical evidence of those positive daily associations are not clear in the literature. More research is needed to understand the temporal dynamic of these two behavioral outcomes. Future research should be specifically conducted in individuals with weight concerns and insomnia symptoms. Indeed, participants included in this study presented very good sleep efficiency ( $90 \%$ on average). Other results could be expected in adults with concomitant overweight or obesity and sleep quality issues [note that the 3 participants (\#678, \#246 and \#742) presenting a significant association between steps and sleep efficiency, did not present notable differences in their descriptive statistics in comparison to the group averages; see supplemental material \#2]. Other indicators of sleep quality such as sleep onset latency (SOL), wake after sleep onset (WASO) or subjective sleep quality might also be analyzed in future studies. Finally, it is also possible that, on average, physical activity, notably step counts, is not a strong determinant of sleep quality and vice-versa comparing to other variables [e.g., daylight exposure for sleep (Tamrat et al., 2014); health status, or self-efficacy for physical activity (Bauman et al., 2012)].

In terms of the strength of the associations, our personspecific analyses highlighted that physical activity and sleep mutually interact with different magnitudes from one participant to another. For example, for participant \#1099 (model 1; Fig. 2), sleep duration appears to be a clinically relevant determinant of physical activity; sleeping one hour more was associated with an average decrease of 1200 steps the following day (20 steps for each minute of sleep), which is equivalent to roughly $10 \%$ of the participants usual daily physical activity level (14 k steps per day on average). While for participant \#296 (model 1; Fig. 2), this effect, albeit significant, was half the size. Sleeping one hour more was associated with an average decrease of around 600 steps the following day ( 10 steps/minute of sleep), which is equivalent to roughly $5 \%$ of the usual daily physical activity level $(11 \mathrm{k}$ steps per day on average for that participant). These differences in the magnitude of the results from one participant to another is relevant from a precision medicine perspective, because it suggests that only some participants might truly benefit from an intervention targeting the conflicting association between steps and sleep duration (i.e., participant \#1099, rather than \#206, might benefit from such an intervention given the magnitude of the effects for the first-mentioned). Given that physical activity and sleep are frequently monitored during weight loss programs, such information 
could directly influence the real-time tailoring of a specific behavioral intervention for some participants (Nahum-Shani et al., 2018).

Finally, although we expected some heterogeneity in the form of these associations, our results highlighted linear instead of non-linear associations between the two behaviors at the individual level (see supplemental material \#4). The absence of non-linearity might be due to a samplingmeasurement issue (i.e., 100 observations per participant is probably insufficient to model true non-linear associations for these outcomes), or the fact that our physical activity and sleep outcomes remained mostly unchanged during the data collection period. A greater variance at the individual level in each outcome might be required to observe some form of non-linearity in those associations. This hypothesis would need to be further explored in the future with a larger dataset, both in terms of sample size and number of observations per individual.

\section{Study strengths and limitations}

The strengths of the present study include $(i)$ the length of the behavioral time series collected and analyzed (i.e., 215 days and nights on average per participants), which is extensively higher than most previous studies analyzing the associations between physical activity and sleep, thus increasing the ecological validity of the present results; (ii) the inclusion of overweight or obese young adults participating in a weight management program (to our knowledge this is only the second study on this topic performed among this population); and (iii) our modelling efforts, including the test of person-specific associations and non-linear associations. Our modelling approach provides original and unique information about the temporal dynamic of physical activity and sleep behaviors at the individual level and relevant information to develop, for instance, just-in-time adaptive interventions integrating the potential associations between sleep and physical activity in their algorithm (Hekler et al., 2018; Nahum-Shani et al., 2018).

The study also has limitations. First, because the Fitbit is accurate to detect sleep-wake states, we focused on two derived outcomes of sleep quantity and quality: total sleep time and sleep efficiency. These two outcomes however cannot provide a full understanding of sleep behavior and other results might be observed with complementary sleep outcomes (i.e., SOL, WASO). Similarly, we did not measure daily subjective sleep quality as part of this study, whereas a previous study has shown significant associations between subjective sleep quality and physical activity at the daily level (Tang \& Sanborn, 2014). This limitation (i.e., having multiple indicators of physical activity) applies less to physical activity, because step count is highly correlated with physical activity intensity (Tudor-Locke et al., 2011) and an overall good indicator of physical activity behavior over other metrics (Chomistek et al., 2017). However, in depth analyses of the type and nature of physical activity (e.g., outdoor versus indoor) or proximity with bed time would have allowed us to draw a different and probably more accurate picture of the physical activity and sleep associations (Kredlow et al., 2015). Second, we mainly focused on the daily associations between the two behaviors at lag -1 (including lag -2 as a control variable), or in other words, the associations between behaviors with the previous night or day. However, more complex patterns of associations could emerge with different time lags. Indeed, accumulation effect, social patterns, or hormonal rhythms occurring in different time scales and might influence the associations between physical activity and sleep (Monk, 2010). Although our ancillary analyses indicate that the strongest associations were observed at lag-1 (notably for model 1; total sleep time-> steps), two participants reported persistent significative associations over time up to lag-6 (see the results section). This suggest that some participants can experience cumulative effects over time (shorter sleep durations in this case) and that only analyzing these associations at lag-1 might impede more precise interpretation of the sleep-physical activity relationships (see on that topic, Irish et al., 2014). New visualization and/or statistical methods are needed to better understand the dynamic patterns of association between sleep and physical activity at different lags (see Hasselman \& Bosman, 2020; Heino et al., 2021 for methods from the field of complexity). Third, in regard to generalizability, results from this study are limited to our participants characteristics, i.e., young adults who are overweight or obese, without comorbidities, participating in a weight management program who generally are physically active and have good sleep efficiency on average, as well as a low sample size (i.e., $N=33$; which is a limitation here in regards of the generalizability of our results, but not in term of statistical power). It would be interesting to study similar associations in adults who are overweight or obese and have concomitant sleep disorders issues. Further, as the trial is still ongoing, group assignment remained unknown for the present study and was not modelled in our analyses, which constitutes a limit given that results might differ by groups (albeit no specific trends in the time-series for each outcome were detected; see supplemental material \#5 at https://osf. io/y7nxg/).

\section{Conclusion}

Results highlighted significant negative and linear personspecific associations between total sleep time and next day 
steps for a majority of the participants included in this study, as well as negative associations between steps and total sleep time the following day for a third of the participants. Few significant person-specific associations were found between physical activity and sleep efficiency. Taken together these results suggest a potential conflicting association between total sleep time and physical activity in the daily lives of some young adults who are overweight or obese.

Author contribution All authors contributed to study conception and design, as well as analysis and interpretation. JG contributed to the data acquisition. GC approved the final version for publication.

Funding The present research was funded by a grant from the National Heart, Lung, and Blood Institute (NHLBI); R01HL136769 attributed to JG. The first author (GC) acknowledges support from the Spanish Ministry of Science and Innovation and State Research Agency through the "Centro de Excelencia Severo Ochoa 2019-2023" Program (CEX2018-000806-S), and support from the Generalitat de Catalunya through the CERCA Program.

\section{Declarations}

Conflict of interest The authors have no conflicts of interest to declare.

Ethics approval All relevant ethical regulations have been followed and participants have completed informed consent.

Human and Animal Rights and Informed Consent All procedures performed in this study involving human participants were conducted in accordance with the ethical standards of the local institutional ethics committee and with the Helsinki Declaration of 1975, as revised in 2000. Informed consent was obtained from all patients for being included in the study.

\section{References}

Ariens, S., Ceulemans, E., \& Adolf, J. K. (2020). Time series analysis of intensive longitudinal data in psychosomatic research: A methodological overview. Journal of Psychosomatic Research, 137, 110191. https://doi.org/10.1016/j.jpsychores.2020.110191

Atoui, S., \& G, C., Romain, A. J., Kingsbury, C., Lachance, J. P., \& Bernard, P. . (2021). Daily associations between sleep and physical activity: A systematic review and meta-analysis. Sleep Medicine Reviews. https://doi.org/10.1016/j.smrv.2021.101426

Baron, K. G., Reid, K. J., \& Zee, P. C. (2013). Exercise to improve sleep in insomnia: Exploration of the bidirectional effects. Journal of Clinical Sleep Medicine: JCSM: Official Publication of the American Academy of Sleep Medicine, 9(8), 819-824. https://doi. org/10.5664/jcsm. 2930

Bauman, A. E., Reis, R. S., Sallis, J. F., Wells, J. C., Loos, R. J., \& Martin, B. W. (2012). Correlates of physical activity: Why are some people physically active and others not? The Lancet, 380(9838), 258-271. https://doi.org/10.1016/S0140-6736(12)60735-1
Bernard, P., Ivers, H., Savard, M.-H., \& Savard, J. (2016). Temporal relationships between sleep and physical activity among breast cancer patients with insomnia. Health Psychology: Official Journal of the Division of Health Psychology, American Psychological Association, 35(12), 1307-1315. https://doi.org/10.1037/hea00 00408

Bernard, P., Doré, I., Romain, A.-J., Hains-Monfette, G., Kingsbury, C., \& Sabiston, C. (2018). Dose response association of objective physical activity with mental health in a representative national sample of adults: A cross-sectional study. PLoS ONE, 13(10), e0204682. https://doi.org/10.1371/journal.pone.0204682

Breheny, P., \& Burchett, W. (2017). Visualization of Regression Models Using visreg. The R Journal, 9(2), 56-71.

Bromley, L. E., Booth, J. N., Kilkus, J. M., Imperial, J. G., \& Penev, P. D. (2012). Sleep Restriction Decreases the Physical Activity of Adults at Risk for Type 2 Diabetes. Sleep, 35(7), 977-984. https:// doi.org/10.5665/sleep. 1964

Brown, T., Avenell, A., Edmunds, L. D., Moore, H., Whittaker, V., Avery, L., \& Summerbell, C. (2009). Systematic review of longterm lifestyle interventions to prevent weight gain and morbidity in adults. Obesity Reviews: An Official Journal of the International Association for the Study of Obesity, 10(6), 627-638. https://doi.org/10.1111/j.1467-789X.2009.00641.x

Burg, M. M., Schwartz, J. E., Kronish, I. M., Diaz, K. M., Alcantara, C., Duer-Hefele, J., \& Davidson, K. W. (2017). Does Stress Result in You Exercising Less? Or Does Exercising Result in You Being Less Stressed? Or Is It Both? Testing the Bi-directional Stress-Exercise Association at the Group and Person (N of 1) Level. Annals of Behavioral Medicine, 51(6), 799-809. https://doi.org/10.1007/s12160-017-9902-4

Cappuccio, F. P., Taggart, F. M., Kandala, N.-B., Currie, A., Peile, E., Stranges, S., \& Miller, M. A. (2008). Meta-Analysis of Short Sleep Duration and Obesity in Children and Adults. Sleep, 31(5), 619-626. https://doi.org/10.1093/sleep/31.5.619

Chaput, J.-P., Després, J.-P., Bouchard, C., \& Tremblay, A. (2008). The Association Between Sleep Duration and Weight Gain in Adults: A 6-Year Prospective Study from the Quebec Family Study. Sleep, 31(4), 517-523. https://doi.org/10.1093/sleep/ 31.4.517

Chaput, J.-P., Dutil, C., Featherstone, R., Ross, R., Giangregorio, L., Saunders, T. J., Janssen, I., Poitras, V. J., Kho, M. E., Ross-White, A., \& Carrier, J. (2020). Sleep duration and health in adults: An overview of systematic reviews. Applied Physiology, Nutrition, and Metabolism, 45(10), S218-S231. https://doi.org/10.1139/ apnm-2020-0034

Chennaoui, M., Arnal, P. J., Sauvet, F., \& Léger, D. (2015). Sleep and exercise: A reciprocal issue? Sleep Medicine Reviews, 20, 59-72. https://doi.org/10.1016/j.smrv.2014.06.008

Chevance, G., Golaszewski, N. M., Baretta, D., Hekler, E. B., Larsen, B. A., Patrick, K., \& Godino, J. (2020a). Modelling multiple health behavior change with network analyses: Results from a one-year study conducted among overweight and obese adults. Journal of Behavioral Medicine. https://doi.org/10.1007/ s10865-020-00137-2

Chevance, G., Perski, O., \& Hekler, E. B. (2020b). Innovative methods for observing and changing complex health behaviors: Four propositions. Translational Behavioral Medicine, 11(2), 676-685. https://doi.org/10.1093/tbm/ibaa026

Chomistek, A. K., Yuan, C., Matthews, C. E., Troiano, R. P., Bowles, H. R., Rood, J., Barnett, J. B., Willett, W. C., Rimm, E. B., \& Bassett, D. R. (2017). Physical Activity Assessment with the ActiGraph GT3X and Doubly Labeled Water. Medicine and Science in Sports and Exercise, 49(9), 1935-1944. https://doi.org/10.1249/ MSS.0000000000001299

Conner, M., Abraham, C., Prestwich, A., Hutter, R., Hallam, J., SykesMuskett, B., Morris, B., \& Hurling, R. (2016). Impact of goal 
priority and goal conflict on the intention-health-behavior relationship: Tests on physical activity and other health behaviors. Health Psychology: Official Journal of the Division of Health Psychology, American Psychological Association, 35(9), 1017-1026. https://doi.org/10.1037/hea0000340

de Zambotti, M., Goldstone, A., Claudatos, S., Colrain, I. M., \& Baker, F. C. (2018). A validation study of Fitbit Charge $2^{\mathrm{TM}}$ compared with polysomnography in adults. Chronobiology International, 35(4), 465-476. https://doi.org/10.1080/07420528.2017.1413578

Fanning, J., Mackenzie, M., Roberts, S., Crato, I., Ehlers, D., \& McAuley, E. (2016). Physical Activity, Mind Wandering, Affect, and Sleep: An Ecological Momentary Assessment. JMIR MHealth and UHealth. https://doi.org/10.2196/mhealth.5855

Feehan, L. M., Geldman, J., Sayre, E. C., Park, C., Ezzat, A. M., Yoo, J. Y., Hamilton, C. B., \& Li, L. C. (2018). Accuracy of Fitbit Devices: Systematic Review and Narrative Syntheses of Quantitative Data. JMIR MHealth and UHealth, 6(8), e10527. https://doi. org/10.2196/10527

Fogelholm, M., Kronholm, E., Kukkonen-Harjula, K., Partonen, T., Partinen, M., \& Härmä, M. (2007). Sleep-related disturbances and physical inactivity are independently associated with obesity in adults. International Journal of Obesity, 31, 1713-1721.

Geller, K., Lippke, S., \& Nigg, C. R. (2017). Future directions of multiple behavior change research. Journal of Behavioral Medicine, 40(1), 194-202. https://doi.org/10.1007/s10865-016-9809-8

Hasselman, F., \& Bosman, A. M. T. (2020). Studying Complex Adaptive Systems With Internal States: A Recurrence Network Approach to the Analysis of Multivariate Time-Series Data Representing Self-Reports of Human Experience. Frontiers in Applied Mathematics and Statistics. https://doi.org/10.3389/fams.2020. 00009

Hastie, T., \& Tibshirani, R. (1986). Generalized Additive Models. Statistical Science, 1(3), 297-310. https://doi.org/10.1214/ss/11770 13604

Heino, M. T. J., Knittle, K., Noone, C., Hasselman, F., \& Hankonen, N. (2021). Studying Behaviour Change Mechanisms under Complexity. Behavioral Sciences, 11(5), 77. https://doi.org/10.3390/ bs 11050077

Hekler, E. B., Rivera, D. E., Martin, C. A., Phatak, S. S., Freigoun, M. T., Korinek, E., Klasnja, P., Adams, M. A., \& Buman, M. P. (2018). Tutorial for Using Control Systems Engineering to Optimize Adaptive Mobile Health Interventions. Journal of Medical Internet Research. https://doi.org/10.2196/jmir.8622

Irish, L. A., Kline, C. E., Rothenberger, S. D., Krafty, R. T., Buysse, D. J., Kravitz, H. M., Bromberger, J. T., Zheng, H., \& Hall, M. H. (2014). A 24-hour Approach to the Study of Health Behaviors: Temporal Relationships between Waking Health Behaviors and Sleep. Annals of Behavioral Medicine : A Publication of the Society of Behavioral Medicine, 47(2), 189-197. https://doi.org/ 10.1007/s12160-013-9533-3

Knittle, K., Heino, M., Marques, M. M., Stenius, M., Beattie, M., Ehbrecht, F., Hagger, M. S., Hardeman, W., \& Hankonen, N. (2020). The compendium of self-enactable techniques to change and self-manage motivation and behaviour v.1.0. Nature Human Behaviour, 4(2), 215-223.

Kredlow, M. A., Capozzoli, M. C., Hearon, B. A., Calkins, A. W., \& Otto, M. W. (2015). The effects of physical activity on sleep: A meta-analytic review. Journal of Behavioral Medicine, 38(3), 427-449. https://doi.org/10.1007/s10865-015-9617-6

Kwasnicka, D., \& Naughton, F. (2019). N-of-1 methods: A practical guide to exploring trajectories of behaviour change and designing precision behaviour change interventions. Psychology of Sport and Exercise. https://doi.org/10.1016/j.psychsport.2019.101570

Lee, I.-M., Djoussé, L., Sesso, H. D., Wang, L., \& Buring, J. E. (2010). Physical activity and weight gain prevention. JAMA, 303(12), 1173-1179. https://doi.org/10.1001/jama.2010.312
Lemmens, V. E. P. P., Oenema, A., Klepp, K. I., Henriksen, H. B., \& Brug, J. (2008). A systematic review of the evidence regarding efficacy of obesity prevention interventions among adults. Obesity Reviews: An Official Journal of the International Association for the Study of Obesity, 9(5), 446-455. https://doi.org/10.1111/j. 1467-789X.2008.00468.x

Liao, Y., Robertson, M. C., Winne, A., Wu, I. H. C., Le, T. A., Balachandran, D. D., \& Basen-Engquist, K. M. (2020). Investigating the within-person relationships between activity levels and sleep duration using Fitbit data. Translational Behavioral Medicine. https://doi.org/10.1093/tbm/ibaa071

Matthews, C. E., Ainsworth, B. E., Thompson, R. W., \& Bassett, D. R. (2002). Sources of variance in daily physical activity levels as measured by an accelerometer. Medicine and Science in Sports and Exercise, 34(8), 1376-1381. https://doi.org/10.1097/00005 768-200208000-00021

Mattingly, S. M., Grover, T., Martinez, G. J., Aledavood, T., RoblesGranda, P., Nies, K., Striegel, A., \& Mark, G. (2021). The effects of seasons and weather on sleep patterns measured through longitudinal multimodal sensing. Npj Digital Medicine, 4(1), 1-15. https://doi.org/10.1038/s41746-021-00435-2

Mead, M. P., Baron, K., Sorby, M., \& Irish, L. A. (2019). Daily Associations Between Sleep and Physical Activity. International Journal of Behavioral Medicine, 26(5), 562-568. https://doi.org/10.1007/ s12529-019-09810-6

Monk, T. H. (2010). Enhancing Circadian Zeitgebers. Sleep, 33(4), 421-422. https://doi.org/10.1093/sleep/33.4.421

Nahum-Shani, I., Smith, S. N., Spring, B. J., Collins, L. M., Witkiewitz, K., Tewari, A., \& Murphy, S. A. (2018). Just-in-Time Adaptive Interventions (JITAIs) in Mobile Health: Key Components and Design Principles for Ongoing Health Behavior Support. Annals of Behavioral Medicine, 52(6), 446-462. https://doi.org/ 10.1007/s12160-016-9830-8

O’Driscoll, R., Turicchi, J., Beaulieu, K., Scott, S., Matu, J., Deighton, K., Finlayson, G., \& Stubbs, J. (2018). How well do activity monitors estimate energy expenditure? A systematic review and metaanalysis of the validity of current technologies. British Journal of Sports Medicine. https://doi.org/10.1136/bjsports-2018-099643

Reed, D. L., \& Sacco, W. P. (2016). Measuring Sleep Efficiency: What Should the Denominator Be? Journal of Clinical Sleep Medicine: JCSM: Official Publication of the American Academy of Sleep Medicine, 12(2), 263-266. https://doi.org/10.5664/jcsm.5498

Shadish, W. R., Zuur, A. F., \& Sullivan, K. J. (2014). Using generalized additive (mixed) models to analyze single case designs. Journal of School Psychology, 52(2), 149-178. https://doi.org/10.1016/j. jsp.2013.11.004

Tamrat, R., Huynh-Le, M.-P., \& Goyal, M. (2014). Non-pharmacologic interventions to improve the sleep of hospitalized patients: A systematic review. Journal of General Internal Medicine, 29(5), 788-795. https://doi.org/10.1007/s11606-013-2640-9

Tang, N. K. Y., \& Sanborn, A. N. (2014). Better quality sleep promotes daytime physical activity in patients with chronic pain? A multilevel analysis of the within-person relationship. PLOS ONE, 9(3), e92158. https://doi.org/10.1371/journal.pone.0092158

Tudor-Locke, C., Craig, C. L., Aoyagi, Y., Bell, R. C., Croteau, K. A., De Bourdeaudhuij, I., Ewald, B., Gardner, A. W., Hatano, Y., Lutes, L. D., Matsudo, S. M., Ramirez-Marrero, F. A., Rogers, L. Q., Rowe, D. A., Schmidt, M. D., Tully, M. A., \& Blair, S. N. (2011). How many steps/day are enough? For older adults and special populations. International Journal of Behavioral Nutrition and Physical Activity, 8(1), 80. https://doi.org/10.1186/ 1479-5868-8-80

Vetter, C. (2020). Circadian disruption: What do we actually mean? The European Journal of Neuroscience, 51(1), 531-550. https:// doi.org/10.1111/ejn.14255 
Vieira, R., McDonald, S., Araújo-Soares, V., Sniehotta, F. F., \& Henderson, R. (2017). Dynamic modelling of n-of-1 data: Powerful and flexible data analytics applied to individualised studies. Health Psychology Review, 11(3), 222-234. https://doi.org/10. 1080/17437199.2017.1343680

Vrieze, S. I. (2012). Model selection and psychological theory: A discussion of the differences between the Akaike Information Criterion (AIC) and the Bayesian Information Criterion (BIC). Psychological Methods, 17(2), 228-243. https://doi.org/10.1037/ a0027127
Wood, S. N. (2017). Generalized Additive Models: An Introduction with R, Second Edition. Chapman and Hall/CRC. https://doi.org/ $10.1201 / 9781315370279$

Publisher's Note Springer Nature remains neutral with regard to jurisdictional claims in published maps and institutional affiliations. 